\title{
High-frequency transmission line transitions
}

\author{
Leonard T. Hall ${ }^{a, b}$, Hedley J. Hansen ${ }^{a, b, c}$, and Derek Abbott ${ }^{a, b}$ \\ ${ }^{a}$ Centre for Biomedical Engineering, The University of Adelaide, SA 5005 Australia \\ ${ }^{b}$ Department of Electrical and Electronic Engineering, The University of Adelaide, SA 5005 \\ Australia \\ ${ }^{c}$ Electronic Warfare \& Radar Division, DSTO, Edinburgh, SA 5111 Australia
}

\begin{abstract}
This paper demonstrates the use of modern electromagnetic simulation software to design and develop a selection of three novel transmission line transitions, for operation at mm-wavelengths, and an improvement in the performance of existing transitions. Specifically, our three case studies analyse (i) a microstrip-to-stripline transition, (ii) an inverted microstrip transition, and (iii) a stripline-to-finline transition. The important concepts are described and the tools available are explained. A number of novel and effective designs are presented as examples.
\end{abstract}

Keywords: Microstrip, Stripline, Vivaldi antennas, Waveguide Transitions, Millimeter waves, Finline

\section{INTRODUCTION}

There are many examples of transitions between two different transmission line types. These are generally implemented by connecting two transmission lines together using a transition to change the shape of the fields, from one line to the other. The transition also provides any impedance transformation needed. If the characteristic impedance of the two lines is identical, and the field shape is similar, the two lines may be simply butted together with reasonable results.

The advent of modern electromagnetic simulators has allowed engineers to quickly and cheaply test and optimise the design of these transitions. These simulators can generate information that can replace the current rules-of-thumb with a rigorous design procedure. This paper presents an alternative approach using microstrip and stripline transitions as examples. We use the High-Frequency Structure Simulator (HFSS) ${ }^{1}$ tool to generate the required results and test the final layouts. Matlab ${ }^{2}$ is used to process the results and generate the designs.

The techniques described allow small-size transitions exhibiting broadband response to be effectively produced. Furthermore, the size and effectiveness of known transmission line transitions are shown to be able to be reduced in size with more effective matching. The waveguide to finline to microstrip line uses an exponential or elliptical finline geometry to implement the first field shaping and impedance transformation. ${ }^{3}$ The length of the transition is made long enough so that the characteristic impedance of the transition is no longer critical. Using the techniques described in this paper it is shown that this transition can implement a broad-band exponential impedance taper over half a wavelength at the lowest frequency of interest, with the shape of the transition dictated by the "finline inside waveguide" transmission line properties. Results are presented that demonstrate an improved frequency response and size.

Further author information: (Send correspondence to L. T. Hall)

L. T. Hall: E-mail: lthall@eleceng.adelaide.edu.au, Telephone: 0402336642

H. J. Hansen: E-mail: hedley.hansen@dsto.defence.gov.au

D. Abbott: E-mail: dabbott@eleceng.adelaide.edu.au 


\section{MICROSTRIP TRANSITIONS}

Designers mostly implement microstrip transitions by simply matching the impedance of the two transmission lines and butting them together. This approach often suffers from poor matching or high radiation losses. This section will show that microstrip transitions exhibit broad-bandwidth and very low losses when both the impedance and the electromagnetic field is matched through the transition.

The "ports only solution", produced by HFSS, allows the user to generate the transmission line characteristic impedances of unusual geometries, with a precision much greater than approximating the capacitance and inductance of a transmission line geometry. Because this algorithm is time-efficient, the characteristic impedance can be generated across a large grid of design parameters. This in turn allows the geometry to vary gradually over the length of the transition, while keeping the characteristic impedance constant. In doing so the electromagnetic fields are gradually shaped to suit the new transmission line.

\subsection{Microstrip to stripline}

With stripline the fields are contained between the upper and lower ground planes as Fig. 1(b) demonstrates. The circuits constructed can therefore be stacked, allowing large circuits to be accommodated in a compact space. The two ground planes make access to the stripline difficult. Microstrip-to-stripline transitions are attractive in this regard because after a transition to microstrip, shown in Fig. 1(a), it is possible to implement surface mount components or coaxial connectors.

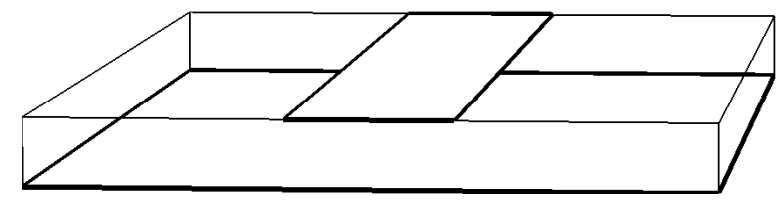

(a) Cross section of the microstrip line.

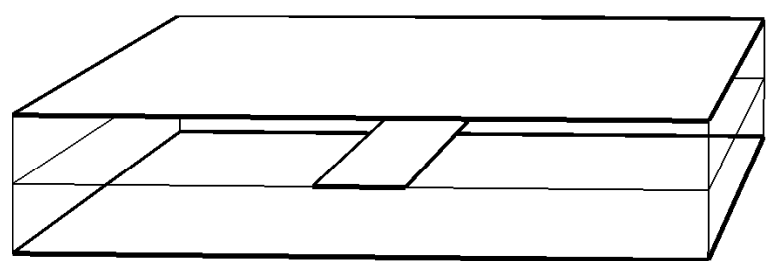

(b) Cross section of the stripline.

Fig. 1. The cross section of the two transmission line types of interest.

In order to expose the stripline and transform the fields to that of a microstrip line, the upper ground plane and substrate must be removed. The stripline is exposed by splitting the upper ground plane and substrate directly above the center of the stripline. To do this in a way that will ensure a smooth field transition, the gap is increased until only the microstrip line remains. Two variables determine the characteristic impedance: line width and gap width. A plot of characteristic impedance for a range of line widths and gap widths is shown in Fig. 2(a). By following the dashed $50 \Omega$ contour line through the transition, constant characteristic impedance is maintained. By assuming a linear increase in upper gap width across the transition length (10 times the substrate height) and calculating the line width required to keep the characteristic impedance constant, the required parameter values, as shown in Fig. 2(b), are generated. The transition layout may then be generated depicted in Fig. 2(c).

Fig. 3 demonstrates how the electric field changes along the length of the transition. Figs. 3(a) and 3(c) show the microstrip line and stripline fields, and Fig. 3(b) shows the fields half way through the transition. The microstrip fields are concentrated between the lower ground plane and line Fig. 3(a). As the gap narrows, the field density increases above the line and the line width must become narrower to keep the characteristic impedance constant Fig. 3(b). Fig. 3(c) shows that as the gap closes the fields become equal in density above and below the line with the line width further reduced to keep the impedance constant.

Fig. 4(a) shows the simulated performance using HFSS. Fig. 4(b) shows a similar estimate if the direct transition, for which both transmission lines have been crudely butted together. The reflection coefficients for 


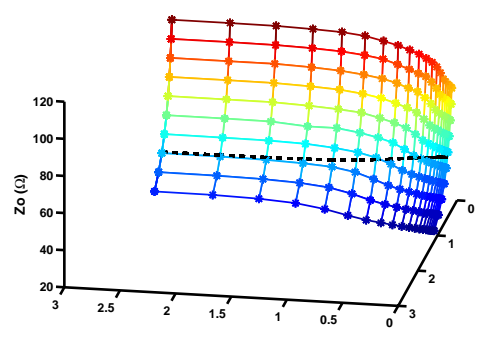

(a) The simulated impedance as the width of the line and upper ground plane gap is varied. The dashed line is the line of constant characteristic impedance of $50 \Omega$.

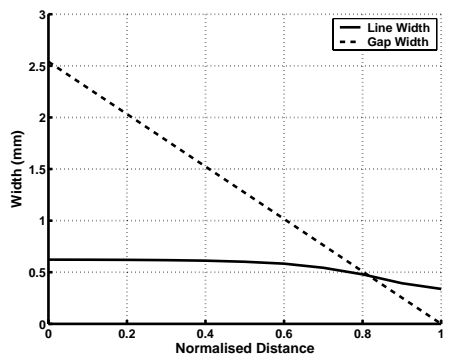

(b) The width of the line and upper ground plane gap through the length of the transition, normalised to the length of the transition, keeping the characteristic impedance constant at $50 \Omega$.

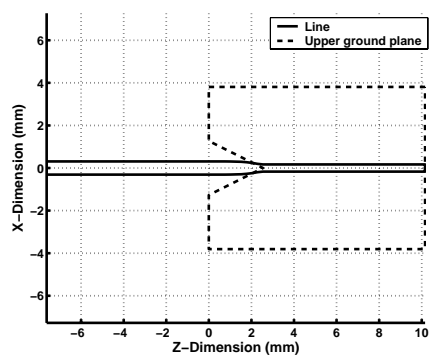

(c) Final layout of the lines and upper ground plane. The lines have a $50 \Omega$ characteristic impedance, 10 mil TLE-95 substrate, $\epsilon=2.3$, with a transition length of 10 substrate heights.

Fig. 2. The simulated results and the resultant microstrip-to-stripline transition design.

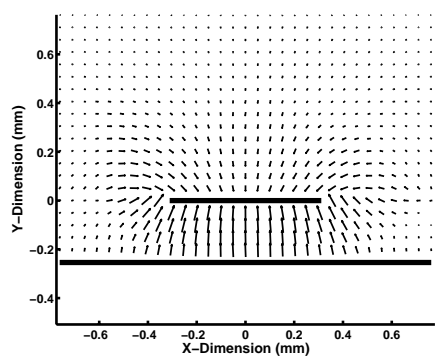

(a) Microstrip electronic field lines.

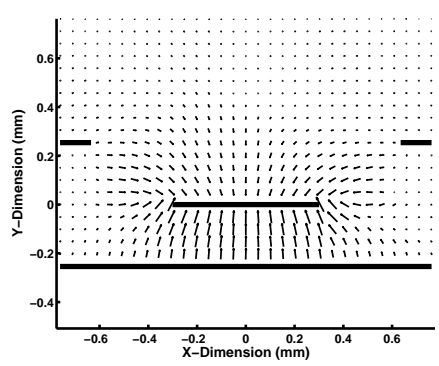

(b) Electronic field lines mid transition.

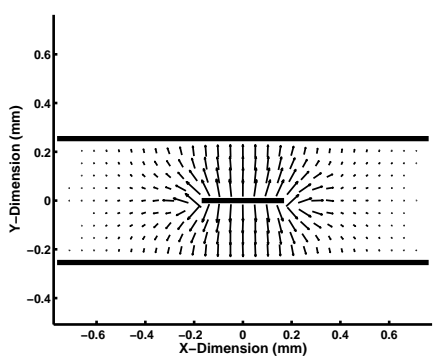

(c) Stripline electronic field lines.

Fig. 3. These three plots show how the electronic field lines gradually vary along the length of the microstrip-to-stripline transition. 
both are quite acceptable, the only substantial improvement is the radiated losses, which are reduced by $3 \mathrm{~dB}$ at $20 \mathrm{GHz}$. The increased radiation losses, for the direct transition, are due to the discontinuity where the line width is stepped from the microstrip width to the stripline width.

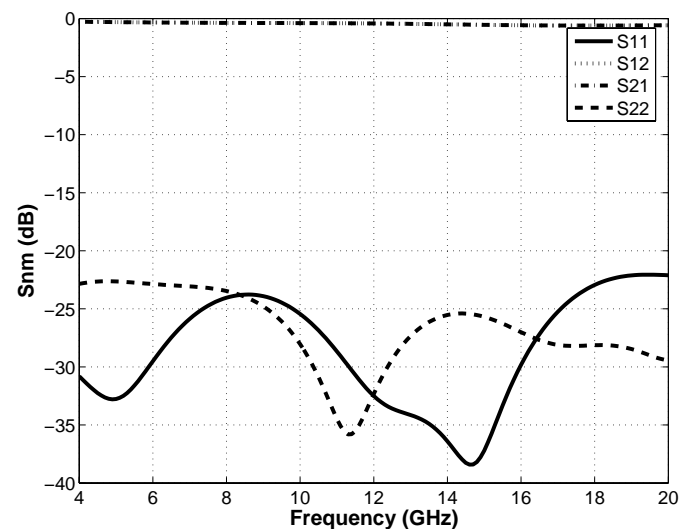

(a) The scattering parameters of the transition shown in Fig. 2(c). Note that $S_{12}$ and $S_{21}$ are equal.

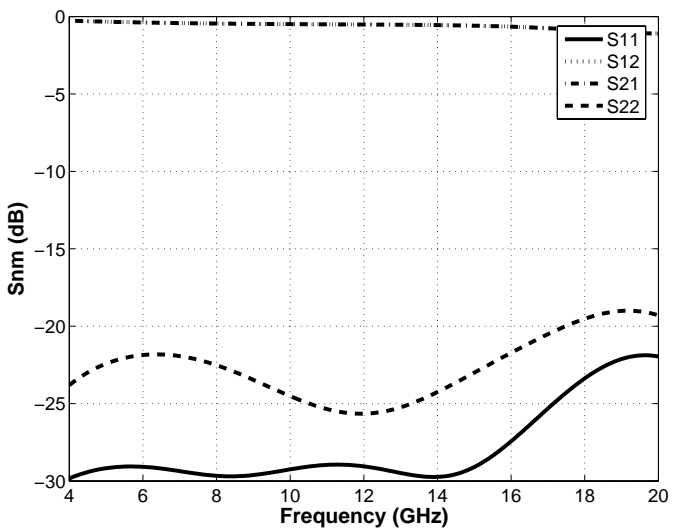

(b) The scattering parameters of the direct transition. Note that $S_{12}$ and $S_{21}$ are equal.

Fig. 4. The frequency dependant scattering parameters of the microstrip-to-stripline transitions, generated using HFSS. This demonstrates a $3 \mathrm{~dB}$ reduction in radiative losses through the transition by suing a gradual transition.

\subsection{Inverted Microstrip}

In applications that require the signal from one side of a ground plane to be transferred to the other side, vias are often used. Vias have substantial parasitic inductance and if substantial losses are to be avoided this inductance must be removed with a matching network.

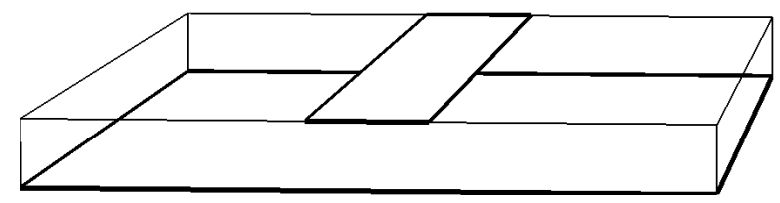

(a) Cross section of the microstrip line.

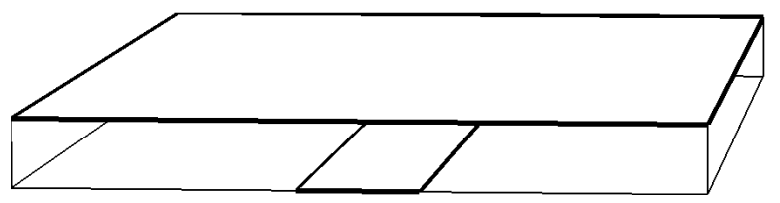

(b) Cross section of the inverted microstrip line.

Fig. 5. The cross section of the two transmission line types of interest.

The inverted microstrip transition swaps the microstrip and ground plane, as shown in Fig. 5, using a parallel microstrip line to join the two sides. The "ports only solution" in HFSS is used to generate the impedance of the parallel microstrip line for a range of upper and lower microstrip widths as seen in Fig. 6(a). The path of constant characteristic impedance of $50 \Omega$ can be determined from the mesh shown in Fig. 6(b). An implementation of the transition for which the characteristic impedance is kept constant over the transition length (10 wavelengths) is shown in Fig. 6(c).

The field pattern is slowly changed along the transition from microstrip, to parallel microstrip lines, to 


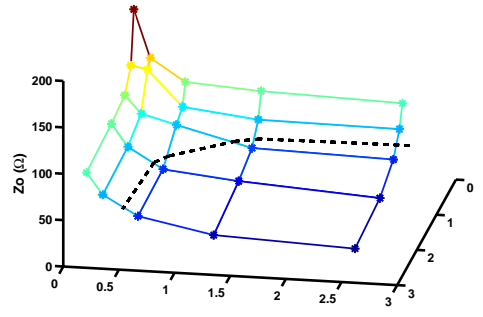

(a) The simulated impedance as the width of the upper and lower line is varied. The dashed line is the line of constant characteristic impedance of $50 \Omega$.

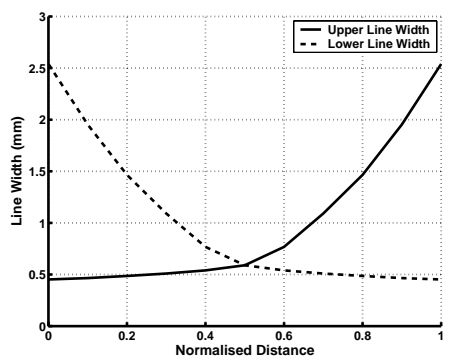

(b) The width of the upper and lower line through the length of the transition, normalised to the length of the transition, keeping the characteristic impedance constant at $50 \Omega$.

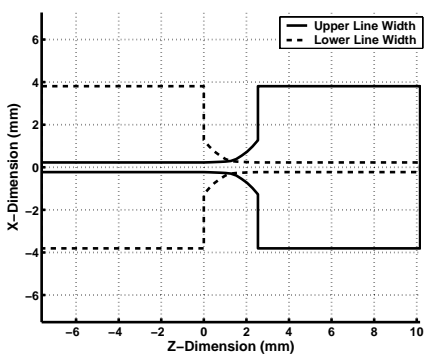

(c) Final layout of the upper and lower microstrip lines. The lines have a $50 \Omega$ characteristic impedance, 10 mil TLE-95 substrate, $\epsilon=2.3$, with a transition length of 10 substrate heights.

Fig. 6. The simulated results and the resultant inverted microstrip transition design.

microstrip again as Fig. 7 demonstrates. This broad-band transition is shown in Fig. 8(a), a very substantial improvement over the direct transition Fig. 8(b).

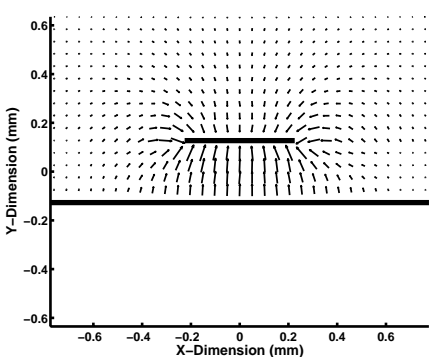

(a) Microstrip electronic field lines.

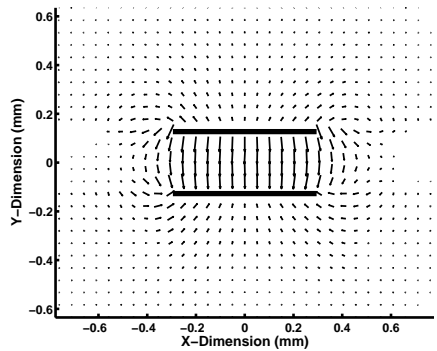

(b) Electronic field lines mid transition.

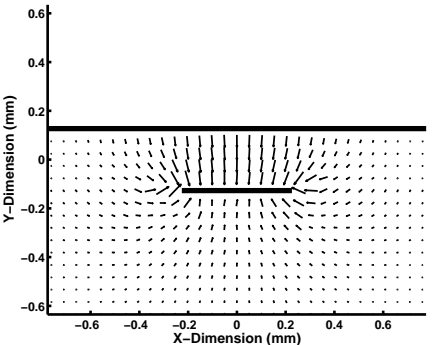

(c) Inverted microstrip electronic field lines.

Fig. 7. These three plots show how the electronic field lines gradually vary along the length of the inverted microstrip transition.

\subsection{Stripline to Balanced Antipodal Finline}

The tapered slot antenna ${ }^{4}$ and Vivaldi antenna have demonstrated very broad-bandwidths. The difficulty in designing these antennas is the feed network that transforms a microstrip or stripline, as seen in Fig. 9(a), feed into a finline, antipodal finline, or balanced antipodal finline, shown in Fig. 9(b). Using the techniques described in this paper we can implement a stripline to balanced antipodal finline feed network that is compact, simple to design, and largely frequency independent.

The first step is to simplify the two transmission line structures into a small number of variables that we may use to generate the transmission line characteristic impedance grid shown in Fig. 10(a). The line width of the stripline is the first and the second must be the overlap of the upper and lower ground plane from the edge of 


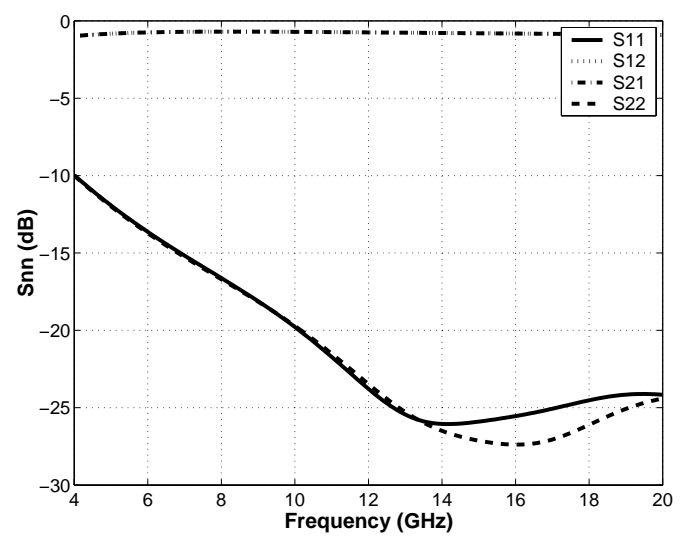

(a) The scattering parameters of the transition shown in Fig. 6(c). Note that $S_{12}$ and $S_{21}$ are equal.

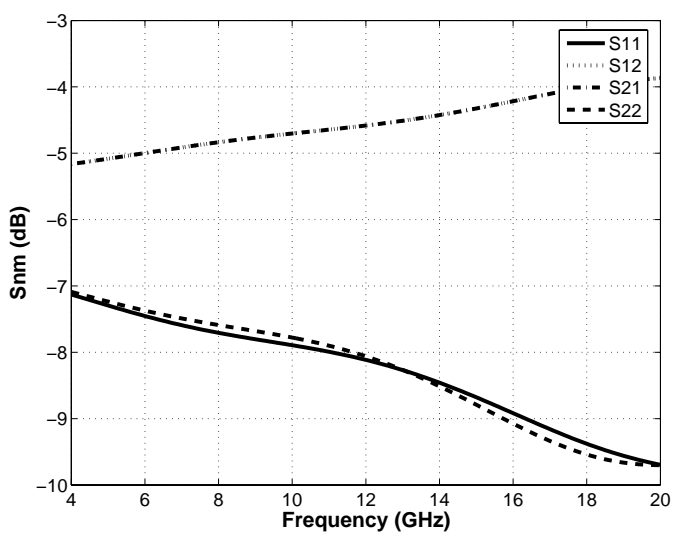

(b) The scattering parameters of the direct transition. Note that $S_{12}$ and $S_{21}$ are equal.

Fig. 8. The frequency dependant scattering parameters of the inverted microstrip transitions, generated using HFSS. This demonstrates a substantial improvement in the performance of the transition.

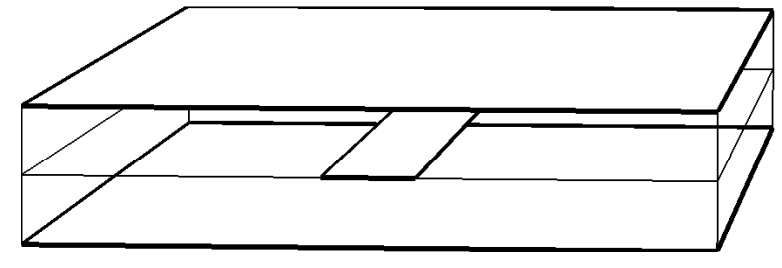

(a) Cross section of the stripline.

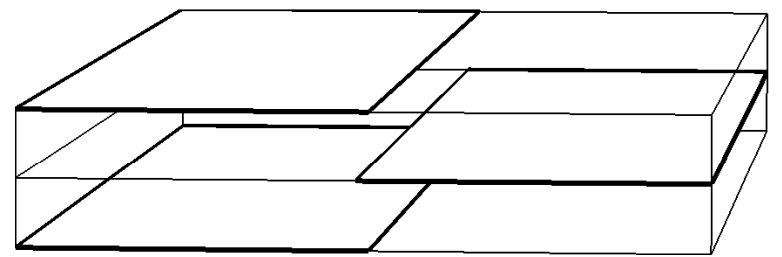

(b) Cross section of the balanced antipodal finline.

Fig. 9. The cross section of the two transmission line types of interest. 
the stripline. By increasing the overlap to infinity, the transmission line becomes a perfect stripline. If the line width is increased to infinity and the overlap becomes close to zero or negative, the transmission line becomes balanced antipodal finline. The relationship between these parameters and characteristic impedance is shown in Fig. 10(a), the line of constant $50 \Omega$ impedance shown by the dashed line. From this data, the line width can be calculated by assuming a circular overlap for the length of the transition, see Fig. 10(b). Finally the layout is generated as seen in Fig. 10(c).

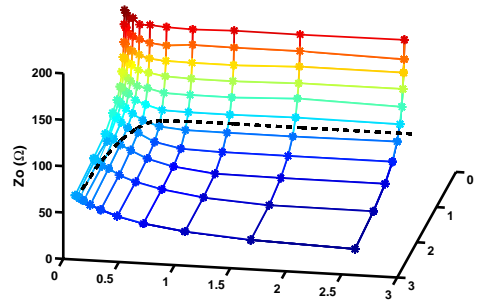

(a) The simulated impedance as the stripline width and ground plane overlap is varied. The dashed line is the line of constant characteristic impedance of $50 \Omega$.

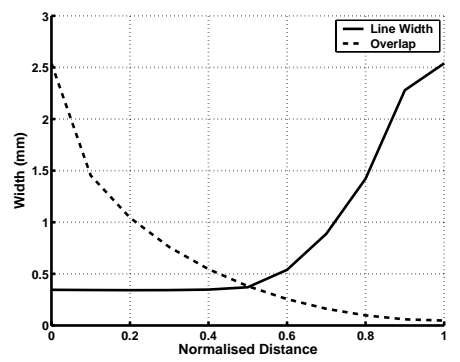

(b) The stripline width and ground plane overlap through the length of the transition, normalised to the length of the transition, keeping the characteristic impedance constant at $50 \Omega$.

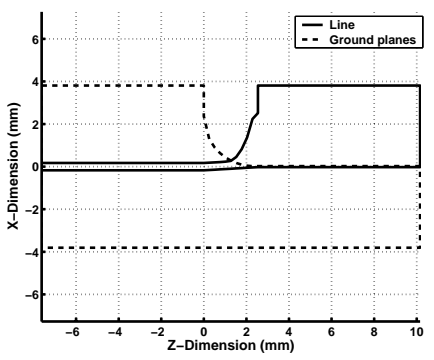

(c) Final layout of the stripline and upper and lower ground planes. The lines have a $50 \Omega$ characteristic impedance, $10 \mathrm{mil}$ TLE-95 substrate, $\epsilon=2.3$, with a transition length of 10 substrate heights.

Fig. 10. The simulated results and the resultant stripline-to-finline transition design.

The field lines gradually change over the length of the transition (10 times the substrate height), as seen in Fig. 11. The microstrip field lines, shown in Fig. 11(a), have the fields pushed to the right by reducing the upper and lower ground plane crossover, as can be seen in Fig. 11(b). As this occurs, the width of the microstrip line is increasing until it becomes a central ground plane and the field lines become that of the balanced antipodal finline Fig. 11(c). The tapered slot or Vivaldi element extends this line by further reducing the overlap, and in doing so increases the characteristic impedance to that of free space.

The performance of this device is shown in Fig. 12(a). The improvement over the direct transition Fig. 12(b) is substantial, offering not only substantially improved match but also much improved insertion losses. This performance may be improved further by selecting the transition shape differently or increasing the length of the transition.

\section{CONCLUSION}

This paper has presented a simple procedure to improve the performance of three known transmission line transitions, as illustrative examples. This new procedure of transition design is applicable to all forms of transmission line transition, including microstrip, stripline, finline, and waveguide. By reducing the number of degrees of freedom in the design, by using this procedure, it is much easier to reproduce a transition at different frequencies or by using different materials.

\section{ACKNOWLEDGMENTS}

Funding from the DSTO RF Hub and the Sir Ross and Sir Keith Smith fund is gratefully acknowledged. 


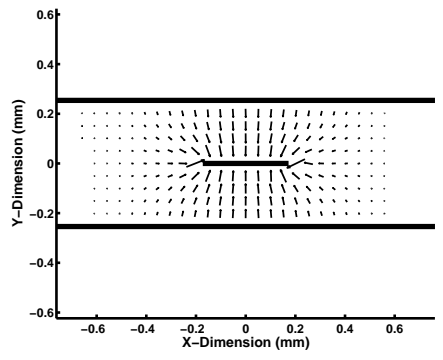

(a) Stripline electronic field lines.

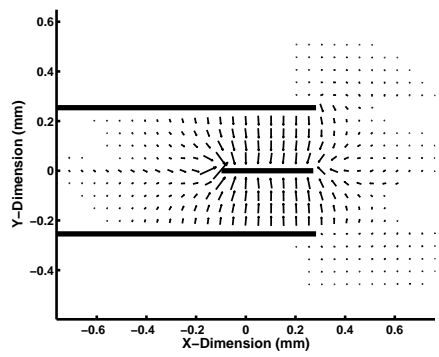

(b) Electronic field lines mid transition.

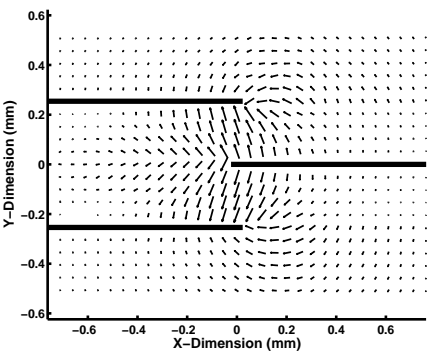

(c) Balanced antipodal finline electronic field lines.

Fig. 11. These three plots show how the electronic field lines gradually vary along the length of the stripline-to-finline transition.

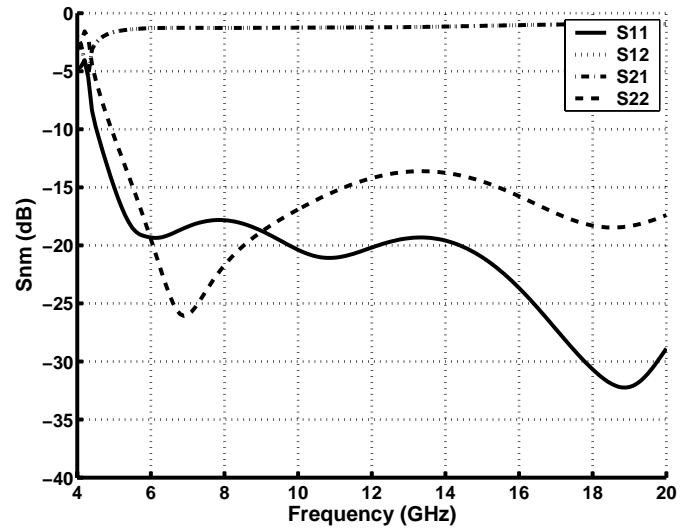

(a) The scattering parameters of the transition shown in Fig. 10(c). Note that $S_{12}$ and $S_{21}$ are equal.

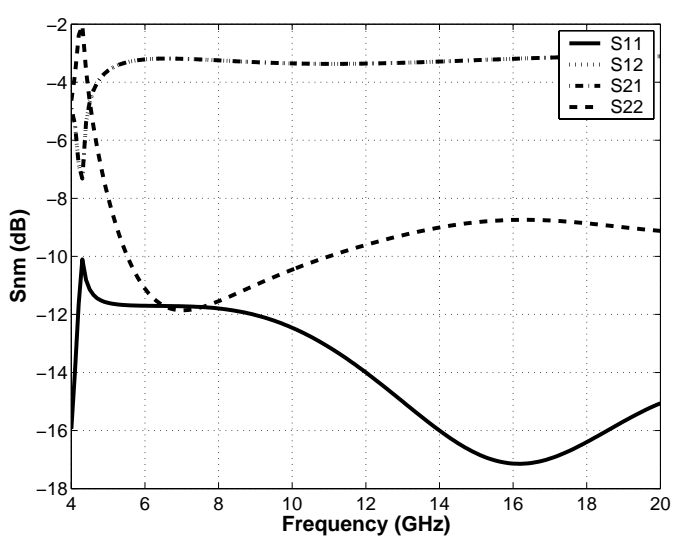

(b) The scattering parameters of the direct transition. Note that $S_{12}$ and $S_{21}$ are equal.

Fig. 12. The frequency dependant scattering parameters of the stripline to balanced antipodal finline transition, generated using HFSS. This demonstrates a substantial improvement in the performance over the direct transition. 


\section{REFERENCES}

1. ANSOFT, "High-frequency structure simulator," 2004.

2. T. MathWorks, "Matlab 6.5.1," 2003.

3. E. K. Sia, J. S. Fu, C. Lu, and S. Tan, "Modeling of antipodal/bcsss transition for millimeter wave finline high q local injected mixer," in Technical Proceedings of the 2001 International Conference on Modeling and Simulation of Microsystems MSM 2001, pp. 522-525, 2001.

4. R. Q. Lee, "Notch antennas," tech. rep., Glenn Research Center, July 2004. 